\title{
Evolution of massive close binaries, compared to observations
}

\author{
Dany Vanbeveren \\ Astrophysical Institute, Vrije Universiteit Brussel, \\ Pleinlaan 2, B-1050 Brussels, Belgium
}

\begin{abstract}
Since binaries can not be considered separately from single stars, we first present a brief summary of massive single star evolution. Next, we highlight massive close binary evolutionary facts that are important when massive close binaries are included in population (number) synthesis. Finally, we consider a few interesting test cases.
\end{abstract}

\section{Introduction}

In the Solar neighbourhood, the binary statistics (binary frequency, orbital parameter distributions, etc.) are still uncertain by at least a factor of two. The situation at larger distances is much worse and the extra-galactic binary population is totally unknown. About one third of the massive OB type stars in the Solar neighbourhood are recognized as the primary star of a close binary. However, most of these binaries have a period smaller than 100 days and mass ratio $q>0.2$. This is obviously due to observational selection and the true binary frequency may be much larger.

Using our experience of massive close binary (MCB) evolution, we have investigated the consequences of MCBs on the interpretation of observations of populations of stars or stellar phenomena in general, starbursts and WR galaxies in particular, and we have to conclude that accounting for the statistical uncertainty, the conclusions resulting from the interpretation of data of populations of stars and/or stellar phenomena where the effects of massive close binaries are omitted, may have an academic value but may be far from reality.

The present state of MCB observations, MCB evolutionary computations and the effect on population synthesis has been discussed in extenso in the monograph The Brightest Binaries (Vanbeveren et al. 1998a) and in two reviews (Vanbeveren et al. 1998b,c). Here, we give a short summary of the evolution of MCBs and we mainly consider the scenarios that we adopt to study the effects of MCB evolution on population (number) synthesis $=\mathrm{P}(\mathrm{N}) \mathrm{S}$. The latter will not be the scope of this paper. We rather discuss a few interesting test cases.

If a star is a component of a close binary, it may encounter a very violent mass loss/mass gain process (the Roche lobe overflow and mass transfer phase) but apart from this, both stars are assumed to evolve as single stars do. Therefore, before discussing the evolution of binary components, we have to consider single star evolution. 


\section{Evolution of massive single stars}

\subsection{The effects of rotation}

How single star evolution can be affected by rotation has been discussed by A. Maeder and N. Langer (these Proceedings). We recall two facts:

$a$. Meridional circulation due to differential rotation of the stellar interior is able to bring nuclear processed matter to the stellar surface within the stellar lifetime;

b. Stellar convective cores may be larger in rotating stars, i.e., the effects of convective core overshooting mimic the effects of rotation.

\subsection{The effect of stellar wind mass loss}

Apart from the stellar wind (SW) mass loss during the OB phase prior to a possible LBV phase (we use the latest results of the Münich group, Puls et al. 1996; Petrenz \& Puls 1996), massive star evolution is critically affected by SW mass loss during the LBV phase, during the RSG phase and during the WR phase. We use the following formalisms:

$a$. The RSG phase: Jura (1987) analyzed 16 RSGs in the LMC. We use a relation which is based on these observations and we assume that these RSG mass loss rates depend on metallicity as predicted by the radiatively driven SW mass loss theory;

$b$. The LBV phase: the $\dot{M}$ during the LBV and RSG phase of a star with $\mathrm{M}_{\text {bol }} \leq-9.5\left(M>40 \mathrm{M}_{\odot}\right)$ must be sufficiently large to assure a RSG phase which is short enough to explain the lack of observed RSGs with $\mathrm{M}_{\mathrm{bol}}<-9.5$. We assume that the SW mass loss during the LBV phase of a star with $M \leq 40 \mathrm{M}_{\odot}$ does not critically affect its evolution;

$c$. The WR phase: we use the values resulting from the atmospherical analysis with inhomogeneous stellar wind models, implying mass loss rates that are a factor 2-3 smaller compared to those derived with homogeneous models (see papers of P.A. Crowther, W.-R. Hamann and D.J. Hillier, these Proceedings).

When we compare our single star models to the standard calculations of Schaller et al. (1992) and Meynet et al. (1994) who are using different SW formalisms during the RSG and WR phases, the following two differences are important:

$d$. due to the RSG mass loss, the $20 \mathrm{M}_{\odot}$ and the $15 \mathrm{M}_{\odot}$ stars lose all their hydrogen rich outer layers and return to the blue part of the HR diagram. For the $15 \mathrm{M}_{\odot}$ star, this happens at the end of its core helium burning $(\mathrm{CHeB})$, i.e., the time spend as a hydrogen deficient $\mathrm{CHeB}$ star is small;

$e$. the final masses at the end of $\mathrm{CHeB}$ are significantly larger than in the standard calculations. Using the post- $\mathrm{CHeB}$ evolutionary computations of Woosley (1986), the corresponding FeNi-core masses indicate that all single stars with initial mass larger than $20-30 \mathrm{M}_{\odot}$ may collapse into a black hole $(\mathrm{BH})$.

\section{Evolution of massive close binaries prior to primary collapse}

Scientists dealing with massive close binary (MCB) evolution always define the primary as the component which was originally the most massive component; 
the originally less massive star is called the secondary. The mass ratio $q$ equals the mass of the secondary divided by the mass of the primary.

\subsection{MCBs with primary mass $>40 \mathrm{M}_{\odot}$}

In these binaries, the LBV stellar wind competes with RLOF and if the former is large enough, the latter is avoided. In this case, the secondary evolution will hardly be affected by mass accretion whereas the period increases. E.g., a $50+30 \mathrm{M}_{\odot}$ binary with an initial period $P=10$ days, will become a $23+29 \mathrm{M}_{\odot}$ WNL+O8III system with a period $P=24$ days.

\subsection{MCBs with primary mass $\leq 40 \mathrm{M}_{\odot}$}

MCBs with mass ratio $q \leq$ 0.2. The classical RLOF has no meaning here. Rather, the most massive component absorbs most of the orbital angular momentum of the smaller mass star and the latter is dragged into the envelope of the former: the process is known as the Spiral-In (SpI) process in binaries with $\mathrm{q} \leq 0.2$. To estimate the evolution of a system like this, one can use the formalism proposed by Webbink (1984). Due to viscosity, orbital energy of the low mass star is transformed into thermal energy of the envelope of the massive component. Part of this is radiated, the other part drives mass loss from the star. The process is very rapid and no accretion is expected onto the star that spirals-in. It can readily be checked that independent of the parameters in the formalism, most of these binaries will merge, i.e., a single star is formed but obviously with a chemistry that differs entirely from the one of a normal star. The evolution of mergers has been investigated quantitatively by Podsiadlowski et al. (1992) who concluded that at the end of their evolution, mergers may explode as blue stars and produce an event like SN 1987A.

The number of MCBs with $q \leq 0.2$ may be significant if one accounts for the statistical study of Hogeveen (1992) and Halbwachs (1987). The existence of low mass X-ray binaries (with a neutron star or black hole component) not belonging to globular cluster provides indirect evidence for the existence of a significant population of MCBs with an extreme mass ratio.

$M C B s$ with mass ratio $q>0.2:$ case $B_{c} / C$. The primary of a MCB with $2<P<10$ yr fills its Roche lobe while it is a RSG. Due to the fact that a RSG has a convective envelope, the RLOF is extremely violent (the RLOF timescale $=$ the dynamical timescale). Due to the adiabatic reaction of a star with a convective envelope, its expansion is larger the larger the mass loss. Therefore, soon after the onset of the RLOF, both components of the binary are surrounded by a common envelope (CE) and the further evolution is governed by the SpI process, much like in systems with $\mathrm{q} \leq 0.2$ (section 3.2.1). However, unlike the latter, case Bc/C binaries may survive the SpI phase. Even more, SW mass loss during the RSG phase may reduce the importance of the CE phase, increasing the survival probability and thus decreasing the merger rate. E.g., a $30+20 \mathrm{M}_{\odot} \mathrm{MCB}$ with initial period $P=700$ days will, after $\mathrm{CE}$, become a $12+20 \mathrm{M}_{\odot} \mathrm{WR}+\mathrm{OB}$ binary with a period of the order of a decade. However, when the initial period $P=1400$ days, before the CE starts, the RSG primary may have lost already quite some hydrogen rich layers due to RSG stellar wind mass loss. After the removal of all hydrogen rich layers, the system becomes a 
$12+20 \mathrm{M}_{\odot} \mathrm{WR}+\mathrm{OB}$ as well but with a period $P$ of a few thousand days. In both cases, the OB companion is not expected to be affected by mass accretion effects.

$M C B s$ with mass ratio $q>0.2$ : case $A / B_{r}$. $\quad$ Defined as MCBs with $P \leq 600-$ 700 days where the primary fills its Roche lobe while it still has a mostly radiative envelope. Detailed computations have been published in numerous papers (a fairly complete list can be found in the three references given in the introduction). The evolution of the primary is well known and is largely independent from the details of the RLOF process. The main question is how much matter lost by the primary (the mass loser) is transferred and accreted by the secondary (the mass gainer or mass accretor). Some scientists like to compare the situation where the RLOF-gasstream encounters a SW of the companion with the colliding winds situation. However, both situations are very different and can not be compared. Of course, before a definite answer can be given, the hydrodynamics have to be investigated.

Matter can leave a binary in the following way: accretion of mass implies stellar expansion and both stars may come into contact. When the contact surface passes through the second Lagrangian point $\left(\mathrm{L}_{2}\right)$ matter leaves the binary and forms a ring. It is fairly easy to calculate the loss of orbital angular momentum in this case and thus to determine the orbital period variation. It is important to realize then that:

The loss of matter from the binary through $L_{2}$ is always accompanied by the loss of a significant fraction of the orbital angular momentum implying an orbital period decrease.

To be able to determine the amount of matter that will leave the binary in this way, it is essential to follow the evolution of both components of a binary simultaneously and to consider the effects of mass accretion on stellar structure in detail. The latter depends on whether the accretion implies large scale mixing (Vanbeveren \& de Loore 1992) or whether accretion happens gently (the standard accretion model, Neo et al. 1977). The way semi-convection is treated is very important in the latter case (Braun \& Langer 1995). In Brussels, we hope to complete these time consuming calculations before the next WR symposium.

\section{Primary core collapse: consequences}

At the end of nuclear burning, the core collapses, either as a $\mathrm{BH}$ or as a neutron star (NS). When a $\mathrm{BH}$ is formed, we adopt the model where the whole mass of the star falls onto the $\mathrm{BH}$ and no supernova explosion happens (Burrows 1987). The formation of a NS is accompanied by a supernova (SN) explosion. Recent pulsar space velocities indicate that the SN explosion is highly asymmetrical (Lorimer et al. 1997). As a consequence, the effects of the SN explosion on the orbital parameters of a MCB must be considered in detail in a $P(N) S$ code, especially when dealing with the expected hard X-radiation in stellar populations where MCBs play an important role. Due to the SN explosion of the primary, the whole binary (in case of non-disruption) or the disrupted OB-type secondary acquires a peculiar space velocity and the OB star can be observed as a runaway (Blaauw 1961); i.e., the runaway nature of a star can be an indication that the star was a former mass gainer of a MCB. 


\section{Evolution of MCBs after primary collapse}

This depends obviously on whether or not the primary exploded as a SN, thus on whether or not the binary was disrupted or not, but depends also on how the structure of the mass gainer was affected due to accretion. We have a library of about 2000 evolutionary sequences where the detailed evolution of the mass gainer in a case $\mathrm{A} / \mathrm{B}_{r}$ binary is followed until its own $\mathrm{CHeB}$ phase, for two accretion modes: the standard gentle accretion model (Neo et al. 1977) and the accretion induced full mixing model (Vanbeveren \& de Loore 1992). When a star has a compact companion (cc), its evolution will be governed by the SpI process.

\subsection{The SpI phase: a CHeB + cc binary or a Thorne-Zytkow object?}

Since an $\mathrm{OB}+\mathrm{cc}$ binary has an extreme mass ratio, its evolution is determined by the SpI process (section 3.2.1) and also here we can use the formalism of Webbink (1994). It is straightforward to prove that most of the OB+cc binaries with orbital period $P<100$ days will merge; however, unlike the systems discussed in section 3.2.1, the SpI process will continue until the cc has reached the center of the star: a Thorne-Zytkow (TZ) object is formed (Thorne \& Zytkow 1977). A few $\mathrm{OB}+\mathrm{cc}$ binaries (those with a massive $\mathrm{BH}$ or those with $P>100$ days) may survive the SpI phase however, and a $\mathrm{CHeB}(\mathrm{WR})+$ cc binary can be formed with a period of the order of few hours or a few days.

\subsection{The evolution of $T Z$ objects}

The structure and evolution of TZ objects have been studied by Biehle (1991) and Cannon et al. (1992). They have the structure of a RSG. Due to SW mass loss, first the hydrogen rich layers will be stripped off. When its mass and SW are large enough, the star may be observed as a 'weird' WR star. According to the authors quoted above, this SW mass loss dominated evolution continues until the whole stellar mass has been blown away and the cc becomes visible again.

\subsection{The formation of $>2 \mathrm{keV} \mathrm{X}$-rays in $\mathrm{OB}$ (or $\mathrm{CHeB})+\mathrm{cc}$ binaries}

We restrict our discussion to wind fed X-radiation; i.e., we do not consider low mass X-ray binaries and $\mathrm{Be} / \mathrm{X}$-ray binaries. In the latter, X-radiation happens when the cc passes through the disk of the Be star. However, the Be phenomenon is not yet fully understood; it may switch on and off and thus the X-radiation may disappear temporarily.

The formation of X-rays when the cc accretes mass from the SW of the OB component has been studied by Davidson \& Ostriker (1973) and by Illarionov \& Sunyaev (1975) (see also Iben et al. 1995). We recall the following two necessary conditions:

$a$. when the cc is a NS supporting a large magnetic field, its spin period must be small enough to allow accretion. Most of the NSs are born with a spin period $<0.1 \mathrm{sec}$ (Bhattacharya et al. 1992) and a magnetic field B which is Gaussian in $\log \mathrm{B}$ with average value $=12.5$. This period does not allow accretion. This means that to determine the moment an $\mathrm{OB}+\mathrm{cc}$ binary will become an X-ray emitter, one needs a model to calculate the variation of the 
spin period of the NS when matter from the SW of the OB star is trapped in the magnetic field of the cc;

$b$. when the cc is a $\mathrm{BH}$, no magnetic field is expected. When the $\mathrm{BH}$ accretes matter from the SW of the optical star, X-radiation will be emitted provided that the angular momentum of the wind matter is large enough to form a disk around the $\mathrm{BH}$. It is fairly straightforward to translate this condition into binary orbit constraints. One concludes that X-radiation will be formed only when the optical mass losing star is close to filling its Roche lobe. In the case of Cyg X1 , it is therefore not a surprise that the optical star is an O-type supergiant (hypergiant?).

\section{Rotation and MCB evolution}

\subsection{The primary (mass loser)}

Due to rotation, the convective core may be larger. Since, after RLOF/CE/SpI, the mass of the mass loser = the mass of the hydrogen (almost) exhausted core, it is clear that: due to rotation the mass of the final mass after RLOF may be larger compared to values resulting from standard non-rotating computations.

\subsection{The secondary (mass gainer)}

Transfer of mass during RLOF is always accompanied by transfer of angular momentum, i.e., a mass gainer spins up. Whether this spin-up process applies for the whole star or is restricted to the outer layers of the gainer, is unclear at present. But if the whole star is affected, rotational mixing may become important for the further evolution. Test computations were presented by Vanbeveren \& de Loore (1994) for the limiting case that rotation (in addition to typical accretion effects) was able to mix the whole star. These calculations were applied then to explain the probable location in the HR diagram of the optical component of the standard high mass X-ray binary Vela X-1 (Vanbeveren et al. 1994).

\section{A few test cases}

\section{1. $\zeta$ Pup: a single star or a former MCB mass gainer?}

The O-type supergiant $\zeta$ Pup is a runaway with a peculiar space velocity of about $65 \mathrm{~km} \mathrm{~s}^{-1}$. A massive star can become a runaway through the SN scenario in a binary or through cluster ejection due to dynamical interaction of massive stars and MCBs (Leonard \& Duncan 1988, 1990). If $\zeta$ Pup was formed as a single star, it is a very young star $(<3$ million yr old). This means that if the cluster ejection mechanism was responsible for the runaway status, it can be expected that along its kinematical path, the remains of the cluster are still there. However, using Hipparcos data, there is no evidence at all for such a massive cluster, so that we are inclined to conclude that $\zeta$ Pup was a former mass gainer of a MCB. The SN explosion of the primary of the binary disrupted the system and gave the star its runaway velocity. It is interesting to remark that $\zeta$ Pup is a rapid rotator. This may be due to a previous accretion process: accretion of matter lost by RLOF is always accompanied by accretion of significant angular momentum, implying a spin-up of the mass gainer. 


\subsection{V444 Cyg: evidence for quasi-conservative evolution at RLOF}

Obviously, both components in a MCB should be on the same isochrone. When we believe (single) star evolution and the spectral type of the O-component in the WR+OB binary V444 Cyg, we have to conclude that the O6 star is significantly younger than the WR star (recall that the masses of both components are very well known). We consider this as evidence for rejuvenation and thus mass transfer has occurred during the RLOF of the primary of the progenitor system.

A direct argument for mass transfer may be the lack of a massive small scale ring around the system. If all mass lost by the primary due to RLOF (in the progenitor system) would have left the binary, we would expect a $10-14 \mathrm{M}_{\odot}$ ring around the binary and, at present, this has not yet been observed. One could consider this as direct evidence that the secondary of the progenitor acted as an efficient vacuum cleaner and that the RLOF was (quasi)-conservative. Of course, the fact that there is no signature yet for a massive ring around V444 Cyg has to be confirmed by more observations.

Let us remark that the spectral type of the O-component in the SMC WR binary AB 7 (WN+O6V, Niemela, these Proceedings) also suggests (quasi)conservative RLOF in the progenitor.

\subsection{HD 50896: a WR+cc binary?}

If the 3.7 day periodic variation in HD 50896 is due to the presence of a cc, why don't we see X-rays? As shown in Vanbeveren et al. (1982) (and later confirmed by Stevens \& Willis 1988), the absorption of $>2 \mathrm{keV}$ radiation in the wind of a WR star is not large enough to explain the lack of X-rays in HD 50896. Two alternatives: (a) the cc is BH. It can easily be checked that in this case the binary period is too large for an accretion disk to form around the $\mathrm{BH}$ (section 5.3) and no X-rays will be emitted; and (b) the cc is a rapidly rotating NS. The pre-SpI progenitor of the WR+NS binary $(P=3.7$ days) must have been an $\mathrm{OB}+\mathrm{NS}$ binary with a period of about 700 days. It can easily be checked that in this case the spin period of the NS hardly changes due to accretion of matter from the SW of the OB star. This means that, if the NS star was born with a small spin period, it still has a small spin period at the moment the OB star becomes a WR type.

\subsection{HD 197406: a WR+BH binary?}

The WR star shows a 4.32 day period variation and if it is due to the presence of a cc, the resulting mass function suggests a $\mathrm{BH}$ component (Drissen et al. 1986). It is fairly easy then to prove that, if the WR star is a normal WR star, the angular momentum of the SW is too small and an accretion disk around the $\mathrm{BH}$ will not form. This may explain the lack of hard X-rays.

\subsection{Cyg X-3: a WR+cc binary?}

Question: is the optical star a normal WR star with a normal WR-like stellar wind? We first notice that, if the cc is a $\mathrm{BH}$, the orbital period is small enough so that the condition for the formation of an accretion disk (and the formation of X-rays) is fulfilled. Next, we combine: the orbital data of the binary including the observed orbital period variation and the mass function (Van Kerkwijk et al. 1992; Schmutz et al. 1996), the X-ray data (Bonnet-Bidaud \& Chardin 1988) 
with the accretion model (Davidson \& Ostriker 1973), a standard SW velocity law and a detailed model for the absorption of $>2 \mathrm{keV} \mathrm{X-rays,} \mathrm{and} \mathrm{we} \mathrm{have} \mathrm{to}$ conclude that (see also Mitra 1996): the optical star in Cyg X-3 is not a normal WR star.

A probable evolutionary scenario is the following: start with a $50+10 \mathrm{M}_{\odot}$ binary. The primary loses its hydrogen rich layers by and LBV type SW in combination with SpI of the $10 \mathrm{M}_{\odot}$ secondary: the system becomes a $20+10 \mathrm{M}_{\odot}$ $\mathrm{WR}+$ early B-type main sequence star. At the end of its evolution, the WR star collapses to form a $8 \mathrm{M}_{\odot} \mathrm{BH}$ (thus no SN). When the $10 \mathrm{M}_{\odot}$ secondary reaches its Roche lobe, the further evolution is governed by the $\mathrm{CE} / \mathrm{SpI}$ process. When most of the hydrogen rich layers of the secondary are removed, we are left with a 2-2.5 $\mathrm{M}_{\odot} \mathrm{CHeB}$ star which may show up (in the IR) as a WR like star.

At the end of $\mathrm{CHeB}$ (during He shell burning), the $\mathrm{CHeB}$ star expands again, reaches its Roche lobe for the second time and starts losing matter. We propose that Cyg X-3 is in this phase. The X-rays are the result of accretion by the $\mathrm{BH}$ of part of this lost matter.

\section{References}

Bhattacharya, D., Wijers, R., Hartman, J., Verbunt, F. 1992, A\&A 254, 198

Biehle, G. 1991, ApJ 380, 167

Blaauw, A. 1961, Bull. Astron. Inst. Netherlands 15, 265

Bonnet-Bidaud, J., Chardin, G. 1988, Physics Reports 170, 325

Braun, H., Langer, N. 1995a, A\&A 297, 483

Braun, H., Langer, N. 1995b, in: K.A. van der Hucht \& P.M. Williams (eds.), WolfRayet Stars: Binaries, Colliding Winds, Evolution, Proc. IAU Symp. No. 163 (Dordrecht: Kluwer), p. 305

Cannon, R., Eggleton, P., Zytkov, A., Podsiadlowski, Ph. 1992, ApJ 386, 206

Davidson, K., Ostriker, J. 1973, ApJ 179, 585.

Drissen, L., Lamontagne, R., Moffat, A.F.J., et al. 1986, ApJ 343, 426

Halbwachs, J. 1987, A\&A 183, 234

Hogeveen, S. 1992, ApSS. 196, 299

Iben, I., Tutukov, A., Yungelson, Y. 1995, ApJS 100, 217

Illarionov, A., Sunyaev, R. 1975, A\&A 39, 185

Jura, M. 1987, ApJ 313, 743

Leonard, P., Duncan, M. 1988, AJ 96, 222

Leonard, P., Duncan, M. 1990, AJ 99, 608

Lorimer, D., Bailes, M., Harrison, P. 1997, MNRAS 289, 592

Meynet, G., Maeder, A., Schaller, G., et al. 1994, A\&AS 103, 97

Neo, S., Miyaji, S., Nomoto, K., Sugimoto, D. 1977, PASJ 29, 249

Petrenz, P., Puls, J. 1996, A\&A 312, 195

Podsiadlowski, Ph., Joss, P., Hsu, J. 1992, ApJ. 391, 246

Popova, E., Tutukov, A., Yungelson, L. 1982, ApSS 88, 55

Puls, J., Kudritzki, R., Herrero, A., et al. 1996, A\&A 305, 171

Schaller, G., Schaerer, D., Meyent, G., Maeder, A. 1992, A\&AS 96, 269

Schmutz, W., Geballe, T., Schild, H. 1996, A\&A 311, L25 
Stevens, I., Willis, A. 1988, MNRAS 234, 783

Thorne, K; Zytkov, A. 1977, ApJ 212, 862

Vanbeveren, D., van Rensbergen, W., de Loore, C. 1982, A\&A 115, 68

Vanbeveren, D., de Loore, C. 1994, A\&A 290, 129

Vanbeveren, D., Herrero, A., Kunze, D., Van Kerkwijk, M. 1994, SSR 66, 395

Vanbeveren, D., van Rensbergen, W., de Loore, C. 1998a, The Brightest Binaries (Dordrecht: Kluwer)

Vanbeveren, D., De Donder, E., Van Bever, J., et al. 1998b, New Astronomy 3, 443

Vanbeveren, D., de Loore, C., van Rensbergen, W. 1998c, The A\&A Review 9, 63

Vereshchagin, S., Kraicheva, Z., Popova, E., et al. 1987, PAZh 13, 63

Vereshchagin, S., Tutukov, A., Yungelson, L., et al. 1988, ApSS 142, 245

Webbink, R. 1984, ApJ 277, 355

Woosley, S.E.: 1986, in: B. Hauck et al. (eds.), Nucleosynthesis and Chemical Evolution, Proc. 16th Saas-Fee Course (Geneva: Geneva Observatory), p. 1

\section{Discussion}

Walborn: (1) I don't understand your ages for the V444 Cyg components. I would assume that the WR component is the core of a more massive star with a shorter lifetime, while the main-sequence lifetime of an $\mathrm{O} 6$ star is at least $4-5 \mathrm{Myr}$, not 2-3. (2) In my poster I show HST-FOS spatially resolved spectroscopy of the compact WR+OB group Brey 73 in the LMC. The turnoff in at about $30 \mathrm{M}_{\odot}$, but there is a peculiar O4III component with exactly twice that mass, which I propose as a merger.

Vanbeveren: No, the age of an $\mathrm{O} 6 \mathrm{~V}$ star is no more than 2-3 Myr, using standard evolutionary models.

Maeder: The main question is to know which fraction of WR stars owe their existence to binary mass transfer. Let us firstly consider the case of the SMC and suppose that there $100 \%$ of WR stars result from binary mass transfer. This corresponds to a WR/O number ratio of 0.01 . However in the Galaxy the WR/O ratio is at least 10 times larger, which suggests that in the Galaxy the fraction of WR stars resulting from mass transfer is quite small, (unless the binary fraction is larger in the Galaxy).

Langer: Your last viewgraph appears to make a testable prediction concerning the global amount of binaries compared to single stars: you obtain a large amount of blue stragglers in your simulated cluster. Is there really any observed cluster which shows such a large amount of blue stragglers?

Vanbeveren: The amount of blue stragglers is not that large compared to the total number of stars in the simulations $(20000)$ and, indeed, there is observational evidence for a class of rejuvenated stars in some clusters (e.g., Vela OB1, LH 9, LH 10, LH 117 and LH 118).

Moffat: You said that OB star in WR+OB binaries are good vacuum cleaners. Haven't you got the tube attached wrong? We see colliding winds in virtually all close WR+OB systems, with no clear evidence for accretion onto the $\mathrm{OB}$ star, i.e., they are blowing matter out of the system.

Vanbeveren: Colliding winds have nothing to do with the collisions of a high density RLOF gas stream and a low density wind. So the answer to your question is: no. 